\title{
Effects of Spirulina platensis on the Immune Status, Inflammatory and Oxidative Markers of HIV Patients on Antiretroviral Therapy in Cameroon
}

\section{Vicky Jocelyne Ama Moor ${ }^{1,2 *}$, Constant Anatole Pieme ${ }^{1}$, Jan René Nkeck $^{1}$, Prosper Cabral Biapa Nya ${ }^{3}$, Georges Ikomey Mondinde ${ }^{1}$, Falmata Amazia ${ }^{1}$, Charles Kouanfack ${ }^{4}$, Marie Claire Okomo Assoumou ${ }^{1}$ and Jeanne Ngogang ${ }^{1}$}

${ }^{1}$ Faculty of Medicine and Biomedical Sciences, University of Yaounde, I, Cameroon

${ }^{2}$ University Teaching Hospital, Yaounde, Cameroon

${ }^{3}$ Faculty of Sciences, University of Dschang, Cameroon

${ }^{4}$ Faculty of Medicine and Pharmaceutical Sciences, University of Dschang,

Cameroon

*Corresponding Author: Vicky Jocelyne Ama Moor, Faculty of Medicine and Biomedical Sciences, University of Yaounde, I, Cameroon.
Received: February 13, 2021

Published: February 25, 2021

(C) All rights are reserved by Vicky Jocelyne

Ama Moor., et al.

\begin{abstract}
Background: Spirulina platensis is traditionally used as a food supplement with a potential strengthening of the immune system, inhibition of the replication of some viruses, and lipid-lowering effect. The aim of this study was to determine its effect on the inflammatory, immunological, and oxidative status of HIV patients on antiretroviral therapy (ART).

Methods: We performed a study on 62 HIV patients on ART (Tenofovir-Lamivudine-Efavirenz) divided into 2 groups. Group I, supplemented with 10 grams of Spirulina platensis daily, and group II only ART. Participants were paired on ART duration and followedup for 6 months from December 2015 to June 2016 at the HIV care unit of the Yaounde Central Hospital and the Cité-Verte Hospital, Yaounde. Each group was sampled for CD4-count, viral load, serum levels of interleukins 6 and 8, TNF $\alpha$ and oxidative markers (malondialdehyde, FRAP, catalase, total peroxides, superoxide dismutase, glutathione peroxidase, reduced glutathione) at the beginning (T0) and the end of the trial (T1). Ethical clearance was obtained from the Cameroon National Ethics Committee for Human Health and Research (Ref 2016/01/699/CE/CNERSH/SP). Statistical analysis was performed using the software SPSS 21.0. The threshold of significance was set at 0.05 .

Results: Mean ages of participants were $35 \pm 8$ and $37 \pm 7$ respectively for group I and II. We observed a significant decrease in viral load in patients receiving spirulina comparing at T0 and T1 ( $p=0.001)$, not observed for participants in group II ( $p>0.05$ ). CD4-counts were unchanged. There was a significant reduction of TNF $\alpha$ levels in group 1 . The levels of pro-oxidative markers were significantly reduced in group 1 while increased in the other group. Antioxidant levels significantly increased in both groups.

Conclusion: Daily supplementation of Spirulina platensis could improve the immune status of HIV patients on ART, and decrease inflammatory and pro-oxidant levels.
\end{abstract}

Keywords: Spirulina platensis; HIV; Antioxidant; Anti-inflammatory; Immunomodulation 


\section{Abbreviations}

ALAT: Alanine Aminotransferase; ART: Antiretroviral Therapy; ASAT: Aspartate Aminotransferase; EDTA: Ethylene Diamine Tetra Acetate; FRAP: Ferric Ion Reducing Antioxidant Power; GPx: Glutathione Peroxidase; HAART: High Active Antiretroviral Therapy; IFCC: International Federation of Clinical Chemistry and Laboratory Medicine; IL6: Interleukin 6; IL8: Interleukin 8; MDA: Malondialdehyde; PCR: Polymerize Chain Reaction; PLVIH: Person Living with HIV; RG: Reduced Glutathione; ROS: Reactive Oxygen Species; SOD: Superoxide Dismutase; TNF Alpha: Tumor Necrosis Factor Alpha

\section{Introduction}

Spirulina is a microscopic, multicellular blue alga from the class Cyanophyceae and the family Oscillatoriaceae [1]. It is a cyanobacterium traditionally used for several hundred years by certain populations (Delpeuch., et al. 1976). Its protein content represents 60 to $70 \%$ of its dry matter [2], making it a higher source of protein than that of cereals (14\%), fish (25\%), soy (35\%), and powdered milk (35\%) [3]. Spirulina is therefore used as a food supplement in certain countries supported by the World Health Organization and the Food and Drug Administration, especially in people living with the Human Immunodeficiency Virus (PLHIV) [4,5].

HIV infection has a negative effect on the immune system and nutritional status. It participates in the destruction of CD4-T cells by cellular apoptosis, involving the production of reactive oxygen species (ROS) [6]. Oxidative stress is also involved in the transcription of HIV genetic material through general immune activation in the presence of inflammatory cytokines (interferon gamma, TNF $\alpha$ ), proteolytic enzymes (myeloperoxidase) and leukotrienes [7]. The rising in ROS modifies by oxidation; proteins, lipids, DNA and cellular carbohydrates [8]. The High Active Anti-Retroviral Therapy (HAART) has improved the quality of life of people living with HIV by slowing the replication of the virus, but it is accompanied by some adverse effects, including toxicity of noble organs, disruption of lipid and carbohydrate metabolism, digestive disorders and lactic acidosis [9].

Nutrition is an important component of comprehensive care for PLHIV, particularly in countries where food resources are limited [10]. PLHIV have an increased need for a balanced diet because poor nutritional status accelerates the progression of AIDS-relat- ed illnesses and could hind the medical response to antiretroviral therapy (ART) all results in the negative socio-economic impacts of HIV. In Africa, there is little work on the combined effects of ART and nutraceuticals. In Cameroon, Ngo Matip., et al. showed that the daily consumption of $10 \mathrm{~g}$ of spirulina powder for 6 months would stimulate the proliferation of CD4 while decreasing the viral load in ART-naive PLHIV $[11,12]$. However, there are no data on the effect of Spirulina platensis on the oxidative, inflammatory and immunological status of Cameroonian patients, carriers of HIV-1 in adjunction with ART. Therefore, we carry out this study with aim to determine the effect of spirulina consumption by HIV-1 patients being on a first regimen of ART on inflammatory and immunological markers, viral load and oxidative stress.

\section{Materials and Methods}

Study framework

We carried out this study from December 2015 to June 2016. The participants were people who gave their consent, were aged 18 years and above, living with HIV type 1, on first line ART including Tenofovir, Lamivudine and Efavirenz since 6 months, and followed at the HIV care unit of the Yaounde Central Hospital or Cité-Verte Hospital, Yaounde. We did not include participants co-infected with viral hepatitis (B or C), and/or suffering from diabetes, or hypertension, or having an opportunistic infection like tuberculosis. Those who did not give their consent and/or presented with any opportunistic infection during the study period were excluded. The sample size was estimated at 78 with Lorentz's formula with the prevalence of HIV in Cameroon at 4.2\% [13], power and error limit respectively set at $95 \%$ and $5 \%$.

\section{Procedure}

Before the beginning of the study, we obtained administrative authorizations from all the study sites and an ethical clearance from the Cameroon National Ethics Committee on Human Health and Research (Ref 2016/01/699/CE/CNERSH/SP). Before being included, all the potential participants were informed on the benefits and risks of the study and the trial was done with respect of ethical principles. Included participants were equally recruited into the two hospitals, defining two groups (I and II), which were randomly designated for spirulina supplementation. They were paired from ART duration. Participants from group I (from the Yaoundé Central Hospital) received the supplementation with 10 grams of Spirulina platensis daily and the protocol of ART (Teno- 
Effects of Spirulina platensis on the Immune Status, Inflammatory and Oxidative Markers of HIV Patients on Antiretroviral Therapy in Cameroon

fovir, Lamivudine and Efavirenz) while those from group II (from Cité-Verte Hospital) only received ART (Tenofovir, Lamivudine and Efavirenz). Each participant was followed-up monthly for 6 months. At each visit, the principal investigator assessed and reinforced the compliance. All the participants were sampled for CD4-count, viral load, serum levels of interleukins 6 and 8 (IL 6 and 8), TNF $\alpha$ and oxidative markers (malondialdehyde, FRAP, catalase, total plasma peroxides (TPP), superoxide dismutase (SOD), glutathione peroxidase (GPx), reduced glutathione) at the beginning (T0) and the end of the trial (T1). The toxicity during the study was evaluated by the dosage of blood urea nitrogen (BUN), serum creatinine and transaminases (Alanine amino transferase (ALAT) and Aspartate aminotransferase (ASAT).

\section{Spirulina platensis collection and administration}

Spirulina platensis was cultivated in a farm located in Nomayos (Yaoundé, Cameroon). The algae were grown inside a water reservoir at pH 10. The culture was collected and biochemically characterized at the Biochemistry Laboratory of the Faculty of Medicine and Biomedical Sciences of the University of Yaounde I (FMBSUY1) and the Institute of Medical Research and Study of Medicinal Plants as previously described [14]. The algae were conserved in small packages of 10 grams and kept in an aluminum bag. Thirty boxes were distributed to each participant every month, at home or during an appointment at the study site. Each patient received his monthly consumption from the hands of the principal investigator or a nurse participating in the study.

\section{Biological analysis}

The biochemical and immunological analysis were carried out in the biochemistry laboratory of the University Teaching Hospital Yaoundé, the laboratories of Immunology and Biochemistry of the FMBS-UY1 and the CIAB-EXACT laboratory in Yaoundé. At times $\mathrm{T} 0$ and $\mathrm{T} 1$, we collected $15 \mathrm{~mL}$ of venous blood, distributed into 3 tubes including one dry and two with Ethylene diamine tetra acetate (EDTA). The dry tubes were centrifuged at $3000 \mathrm{rpm}$ for 5 minutes. Inflammatory markers, BUN, creatinine and transaminases were measured on the serum obtained. One blood sample containing EDTA was used for the CD4 count and the plasma was used for viral load quantification. The second EDTA tube was used for the assay of oxidative stress markers. The dosage of Blood Urea Nitrogen (BUN) and creatinine were done respectively by the methods of Urease and Jaffé's kinetic test. Transaminases' activity was evaluated by the kinetic method described by IFCC (1986). The cytokine assay (Interleukins 6 and 8, TNF alpha) was performed by the Enzyme Linked Immuno-Sorbent Assay named ELISA-sandwich. The determination of CD4 count was done by flow cytometry using the FACS Count Becton Dickinson system ${ }^{\circledR}$. The Viral load was determined by Real Time Polymerase Chain Reaction (PCR) using the Real Time Amplicor 1.5 and the commercial kit for viral load (Cobas Amplicor - Roche Diagnostics UK ${ }^{\circledR}$ ). The determination of Malondialdehyde (MDA) was done using the method described by Wilbur., et al. (1949). Reduced Glutathione Assay quantification was done using Ellman's technique (1959). The dosage of Catalase activity was made according to the method described by Sinha (1972). The dosage of Glutathione Peroxidase activity was based on the procedure described by Sigma Aldrich. The determination of Superoxide dismutase activity was done by the method described by Misra and Fridovich (1972). The Total Plasma Peroxides (TPP) were determined using the FOX-1 method described by Galli (2005), and the concentration of FRAP was determine with the method described by Benzi and Strain (1996).

\section{Statistical analysis}

The data were analyzed in SPSS (Statistical Package for the Social Sciences) version 21.0. The results are expressed as mean and standard deviation for quantitative variables or median with interquartile interval [Q25; Q75] when data did not show normal distribution, while qualitative variables are presented with effectives and their proportions. The means were compared using the student T-test, while the median were compared using the Median test. Proportions were compared using Fischer exact test. The threshold of significance was set at 0.05 .

\section{Results and Discussion}

Results

Baseline characteristics of the sample

Eighty-six (86) participants were enrolled for the study. From this sample, 21 were lost during follow-up and 3 withdrew their consent. The final sample included 62 participants, equally distributed in both hospitals/groups. The mean ages of participants were $35 \pm 8$ years for group I and $38 \pm 15$ years for group II. The gender distribution was $21(68 \%)$ and 19 (61\%) females respectively for group I and II. At inclusion in the study, the levels of IL6 were significantly higher for participants in group 2. On the comparison of oxidative stress markers in both groups at baseline, the concentra- 
Effects of Spirulina platensis on the Immune Status, Inflammatory and Oxidative Markers of HIV Patients on Antiretroviral Therapy in Cameroon

tions of Reduced Glutathione and FRAP were significantly high in group 1, while total plasma peroxide was significantly low in the same group. Serum levels of Malondialdehyde, superoxide dismutase, glutathione peroxidase, TNF alpha and interleukin 8 did not significantly differ in both groups. The baseline characteristics of both groups including immunological, viral and oxidative markers are summarized in table 1.

Table 1: Baseline characteristics of the study sample.

\begin{tabular}{|c|c|c|c|}
\hline Variables & $\begin{array}{l}\text { Group } 1 \\
(n=31)\end{array}$ & $\begin{array}{l}\text { Group } 2 \\
(n=31)\end{array}$ & $\begin{array}{c}\mathbf{p} \\
\text { value }\end{array}$ \\
\hline Female, n (\%) & $21(68)$ & $19(61)$ & $>0.05$ \\
\hline Age (years) & $35(8)$ & $38(15)$ & $>0.05$ \\
\hline CD4 (cells $/ \mathrm{mm}^{3}$ ) & $443[332 ; 626]$ & $\begin{array}{c}397[321 ; \\
497]\end{array}$ & $>0.05$ \\
\hline $\begin{array}{l}\text { Viral load (copies/ } \\
\mathrm{mL} \text { ) }\end{array}$ & $\begin{array}{c}4900[1250 ; \\
9500]\end{array}$ & $\begin{array}{c}4820[2550 ; \\
8240]\end{array}$ & $>0.05$ \\
\hline $\begin{array}{l}\text { Interleukin } 6 \text { (pg/ } \\
\mathrm{mL})\end{array}$ & $1.56[0.78 ; 2.66]$ & $\begin{array}{c}2.5[2.19 ; \\
3.13]\end{array}$ & 0.03 \\
\hline $\begin{array}{l}\text { Interleukin } 8 \text { (pg/ } \\
\mathrm{mL})\end{array}$ & $\begin{array}{c}23.44 \text { [20.31; } \\
\text { 28.13] }\end{array}$ & $\begin{array}{c}21.88 \text { [20.31; } \\
28.13]\end{array}$ & $>0.05$ \\
\hline $\begin{array}{l}\text { TNF alpha (pg/ } \\
\mathrm{mL})\end{array}$ & $\begin{array}{c}46.24[28.72 ; \\
69.50]\end{array}$ & $\begin{array}{c}57.52[42.52 ; \\
78.76]\end{array}$ & $>0.05$ \\
\hline $\begin{array}{l}\text { Malondialdehyde } \\
(\mu \mathrm{M})\end{array}$ & $2.12[1.6 ; 3.01]$ & $\begin{array}{c}1.67[0.00 ; \\
2.56]\end{array}$ & $>0.05$ \\
\hline $\operatorname{FRAP}(\mu \mathrm{M})$ & $387[355 ; 456]$ & $\begin{array}{c}319[0.00 ; \\
384]\end{array}$ & 0.01 \\
\hline Catalase $(\mu \mathrm{M} / \mathrm{mn})$ & $17.9[13.7 ; 21.5]$ & $\begin{array}{c}16.4[0.00 ; \\
22.9]\end{array}$ & $>0.05$ \\
\hline $\begin{array}{l}\text { Total plasma } \\
\text { peroxides }(\mu \mathrm{M})\end{array}$ & $17.5[15 ; 26.25]$ & $\begin{array}{c}41.25[0.00 ; \\
50]\end{array}$ & 0.04 \\
\hline $\begin{array}{l}\text { Superoxide } \\
\text { dismutase(UI/ } \\
\mathrm{mL} \text { ) }\end{array}$ & $2.37[0.9 ; 5.75]$ & $\begin{array}{c}1.29[0.00 ; \\
5.64]\end{array}$ & $>0.05$ \\
\hline $\begin{array}{l}\text { Glutathione } \\
\text { peroxidase (UI/ } \\
\mathrm{mL} \text { ) }\end{array}$ & $0.82[0.72 ; 0.97]$ & $\begin{array}{l}0.61[0.0 \\
1.04]\end{array}$ & $>0.05$ \\
\hline $\begin{array}{l}\text { Reduced } \\
\text { Glutathione }(\mu \mathrm{M})\end{array}$ & $11.8[8.8 ; 14.7]$ & $\begin{array}{c}9.9[0.00 ; \\
14.3]\end{array}$ & 0.01 \\
\hline
\end{tabular}

FRAP: Ferric ion reducing antioxidant power; TNF alpha: Tumor necrosis factor alpha.

\section{Effects of spirulina platensis}

The study of the different effects of spirulina on the explored parameters is summarized in table 2 . It reveals a significant decrease on the viral load in the supplemented group $(\mathrm{p}=0.001)$ which was not observed in the other group ( $p>0.05$ ). The CD4 count was statistically unchanged both for group 1 and 2 . The concentrations of interleukins 6 and 8 were not statistically different with the consumption of spirulina, however we noticed a slight decrease for IL6 in the non-supplemented group ( $\mathrm{p}=0.01$ ), and a significant reduction for TNF alpha in group 1 which receives daily spirulina $(p=0.04)$. Pro-oxidative makers such as MDA was reduced with the consumption of spirulina ( $p=0.001)$ and increased in the non-supplemented group ( $P=0.004)$, similarly to the activity of FRAP ( $p$ $=0.01$ ). Activities of most of the antioxidants (Catalase, TPP, GPx) were increased in both groups. However, we observed a significant reduction on the activity of the SOD in the two groups. The concentrations of reduced glutathione were statistically unchanged. The exploration of liver and kidney functions showed a significant decrease in the activity of ASAT in both groups $(\mathrm{p}<0.05)$ and ALAT only in group $2(\mathrm{p}=0.009)$. Serum creatinine did not sufficiently vary with the intervention.

\section{Discussion}

HIV infection and its treatment are responsible for many adverse effects on various cells metabolism, which expose the people living with it to many complications. In Sub-Saharan Africa and Cameroon, HIV epidemics continue to cause several deaths. The purpose of this study was to determine the effect of daily supplementation of spirulina in a population of PLHIV already on ART. At six months of supplementation, we observed major effects on the viral load, TNF alpha levels and benefits on the oxidative status with no adverse effects. From this results, we propose that daily supplementation of spirulina could be considerate as a nutritional complement for PLHIV.

This study has involved eighty-six participants, but we encountered 27.9\% loss, more than Ngo Matip., et al. (2015) in Cameroon (17.53\%) and in the Central African Republic (CAR) $(20 \%)[12,15]$. However, we differ from these studies with the inclusion of ARTnaïve patients which are as compliant as those already on ART. The amount of daily supplemented spirulina was set as 10 grams in the course of previous studies [12,15]. The benefits of spirulina on the viral load, the immune system and oxidative status have been 
Effects of Spirulina platensis on the Immune Status, Inflammatory and Oxidative Markers of HIV Patients on Antiretroviral Therapy in Cameroon

Table 2: Evolution of biological parameters.

\begin{tabular}{|c|c|c|c|c|c|c|}
\hline Variables & \multicolumn{3}{|c|}{$\begin{array}{l}\text { Group } 1 \\
(n=31)\end{array}$} & \multicolumn{3}{|c|}{$\begin{array}{l}\text { Group } 2 \\
(n=31)\end{array}$} \\
\hline ASAT (UI/mL) & $30[25 ; 42]$ & $23[18 ; 33]$ & 0.002 & $29[26 ; 38]$ & $26[18 ; 37]$ & 0.001 \\
\hline ALAT (UI/mL) & $20[15 ; 35]$ & $19[9 ; 30]$ & $>0.05$ & $23[17 ; 39]$ & $18[14 ; 30]$ & 0.009 \\
\hline CD4 (cells $/ \mathrm{mm}^{3}$ ) & $443[332 ; 626]$ & $504[395 ; 609]$ & $>0.05$ & 397 [321; 497] & $401[272 ; 492]$ & $>0.05$ \\
\hline Viral load (copies/mL) & $\begin{array}{c}4900[1250 ; \\
9500]\end{array}$ & $440[0.00 ; 3900]$ & 0.001 & $\begin{array}{c}4820[2550 ; \\
8240]\end{array}$ & $\begin{array}{c}4260[2120 ; \\
7420]\end{array}$ & $>0.05$ \\
\hline Interleukin $6(\mathrm{pg} / \mathrm{mL})$ & $1.56[0.78 ; 2.66]$ & $2.18[1.56 ; 2.18]$ & $>0.05$ & 2.5 [2.19; 3.13] & $2.18[1.56 ; 2.65]$ & 0.01 \\
\hline $\operatorname{MDA}(\mu \mathrm{M})$ & $2.12[1.6 ; 3.01]$ & $1.15[0.9 ; 1.41]$ & 0.001 & $\begin{array}{c}1.67[0.00 ; \\
2.56]\end{array}$ & $2.56[0.00 ; 4.1]$ & 0.004 \\
\hline FRAP $(\mu \mathrm{M})$ & $387[355 ; 456]$ & $416[347 ; 520]$ & $>0.05$ & $319[0.00 ; 384]$ & $390[0.00 ; 516]$ & 0.01 \\
\hline Catalase $(\mu \mathrm{M} / \mathrm{mn})$ & $17.9[13.7 ; 21.5]$ & $32.6[22.1 ; 53.7]$ & $<0.001$ & $\begin{array}{c}16.4[0.00 \\
22.9]\end{array}$ & $24.6[0.00 ; 54.2]$ & 0.003 \\
\hline $\mathrm{TPP}(\mu \mathrm{M})$ & $17.5[15 ; 26.25]$ & $35[28.75 ; 42.5]$ & $<0.001$ & $41.25[0.00 ; 50]$ & $32.5[0.00 ; 41.25]$ & 0.01 \\
\hline SOD (UI/mL) & $2.37[0.9 ; 5.75]$ & $1.2[1.13 ; 1.36]$ & $<0.001$ & $\begin{array}{c}1.29[0.00 \\
5.64]\end{array}$ & $0.82[0.00 ; 1.13]$ & 0.001 \\
\hline
\end{tabular}

ALAT: Alanine aminotransferase; ASAT: Aspartate aminotransferase; FRAP: Ferric ion reducing antioxidant power; GPx: Glutathione peroxidase; IL6: Interleukin 6; IL8: Interleukin 8; MDA: Malondialdehyde; RG: Reduced glutathione; SOD: Superoxide dismutase; TNF alpha: Tumor necrosis factor alpha.

previously reported by some authors. In fact, it is supported that Spirulina platensis and ART have anti-inflammatory effects. Experiments conducted in vitro and in the animal model suggest that Spirulina platensis and its components act as immune-stimulators for activating monocytes and macrophages, as well as anti-inflammatory and interferon-producing antibodies [16].

Data from literature show the in vivo antioxidant and antiinflammatory properties of Spirulina platensis. These properties could be related to its components like phycocyanin C. In mice, doses of $100 \mathrm{mg} / \mathrm{kg}$ to $200 \mathrm{mg} / \mathrm{kg}$ of phycocyanin $\mathrm{C}$ were found to be a selective inhibitor of cyclooxygenase -2 (COX-2), and prevent the formation of B4 leukotrienes. An inhibitory concentration of $180 \mathrm{nM}$ for phycocyanin was found, lighter than celecoxib (255nM), and rofecoxib (401nM), which are commercial COX-2 inhibitory anti-inflammatory drugs [17]. Phycocyanin is therefore 2 times more effective than two other anti-COX2 on the market of anti-inflammatories. It would also be a direct inhibitor of 5-lipoxy- 
genase in vitro. In addition, by inhibiting histamine release by rat mast cells, it inhibits the allergic inflammatory response. Phycocyanin also has an anti-hyperalgic effect by decreasing inflammatory nociception by inhibiting nitric oxide (NO), prostaglandin E, COX2 and TNF $\alpha$; by decreasing the inflammation, the pain decreases. Spirulina platensis traps free radicals $\mathrm{COH}^{-}$and $\mathrm{RO} \mathrm{O}^{-}$, reactive oxygen species) during the inflammatory process. Numerous preclinical studies have been conducted on the evaluation of antioxidant and/or anti-inflammatory activities of spirulina in humans. A clinical study in healthy men revealed that oral supplementation for 3 months increased interferon production and activation of natural killer cells (natural killer or NK) [18]. In a study with 26 elderly women, spirulina at a dose of $7.5 \mathrm{~g} /$ day for 8 weeks shows a significant decrease in serum IL- 6 levels and IL- 6 production by antiinflammatory lymphocytes. In another clinical trial of 37 patients with type 2 diabetes, ingestion of spirulina at a dose of $8 \mathrm{~g} /$ day for 12 weeks on the examination for significant reduction of interleukin-6 (IL -6) and malondialdehyde [19].

Phycocyanin does explain all the effects of spirulina. Another compound, Selenium, is a modulator of immune (including antiviral) and anti-inflammatory responses. It participates in the detoxification of certain toxic, heavy and xenobiotic compounds [20]. The antioxidant properties of polyphenols have long been considered as key. Many studies were then carried out on polyphenols and their metabolites and showed their roles as modulators of inflammation signaling pathways. Studies in men following a diet rich in fruits and vegetables were inversely correlated with markers of inflammation (CRP, IL-6) in plasma [21]. In vitro and in vivo studies have shown that polyphenols tend to influence the enzymatic activities of arachidonic acid metabolism, phospholipase A2, cyclooxygenase and lipoxygenase. In vitro studies have also shown that flavonoids such as luteolin or apigenin are inhibited by the production of cytokines such as IL-4, IL-5 and IL-13, that quercetin is inhibited by the production of TNF- $\alpha$ by lipopolysaccharide-stimulated macrophages (LPS), kaempferol inhibited the expression and secretion of TNF- $\alpha$, IL-1 $\beta$ or IL-6 in mast cells [22].

In our study, the consumption of Spirulina for 6 months significantly reduced the level of MDA (prooxidant) who's process of Lipid peroxidation leads to a significant increase in antioxidants. The antioxidant activity of Spirulina is due to its composition, rich in free radical scavengers (vitamins E, $\beta$-carotene, unsaturated lipids, and phenolic acids); antioxidant compounds such as zinc, manga- nese, selenium and copper. These different minerals are cofactors of antioxidant enzymes such as superoxide dismutase, catalase; this could explain the significant increase in these enzymes in patients who have been supplemented with spirulina. The phycocyanin pigment responsible for the blue-green color of this alga has the ability to trap free radicals. It reduces the production of nitrite and the formation of microsomal lipids in the liver responsible for lipid peroxidation [23-25]. In addition, when micronutrients such as selenium (Se) or tellurium (Te) bind to phycocyanin, its antioxidant activity is significantly increased [26]. Several studies have elucidated the signal transduction pathways involved in the antioxidant and anti-inflammatory effects of Spirulina. One study has shown that phycocyanin rapidly stimulates the phosphorylation of signaling molecules related to inflammation such as ERK, JNK, p38 and IKB [27].

Our results are promising but should be interpreted with caution because of the small sample size, the fact that biological measures were not repeated several times knowing that the concentrations could vary on time, and the impossibility for us to measure serum levels of spirulina compounds to verify compliance. Despite these limitations, this study is a base for further research for spirulina.

\section{Conclusion}

The daily consumption of 10 grams of spirulina could improve the immune status through a decrease in viral load. It decreases pro-inflammatory cytokines and improves the oxidative status of HIV patients on ART.

\section{Acknowledgements}

We acknowledge the contribution of Parfait Assoumou, Giovany Zebaze, Nkenfou Blandine, Nsoa Nsoa Junior for the recruitment of participants, and Mr Elague for improving the English language.

\section{Conflict of Interest}

The authors declare there is no competing interest.

This research did not receive funds from any organization.

\section{Bibliography}

1. Barry M., et al. "Intérêt thérapeutique de la spiruline chez l'homme: revue general". International Journal of Biological and Chemical Sciences 8.6 (2014): 2740-2749. 
2. Spiruline. "Technique, pratique et promesse - Ripley Fox" (2019).

3. Henrikson R. "Microalga spirulina : superalimento del future". Urano (1994).

4. IIMSAM NS for. IIMSAM - IIMSAM Spirulina Resource Centre (2019).

5. Food Drug Administration. "Rules and Regulations". 79.10 (2014): 10093-10098.

6. Rabaud C., et al. "Radicaux libres et infection par le VIH". Annales de Biologie Clinique (Paris) 55.6 (1997): 565-571.

7. Repetto M., et al. "Oxidative stress in blood of HIV infected patients". Clinica Chimica Acta 255.2 (1996): 107-117.

8. Montagnier L. Stress oxydant et SIDA (2004).

9. Mamiafo CT., et al. "Hyperlactatemia in a group of HIV patients living in Yaounde-Cameroon". AIDS Research and Therapy 11 (2014): 2.

10. Piwoz E. Nutrition and HIV/AIDS: Evidence, Gaps and Priority Actions. 200449 (190-195).

11. Ngo-Matip M-E., et al. "Effects of Spirulina platensis supplementation on lipid profile in HIV-infected antiretroviral naïve patients in Yaounde-Cameroon: a randomized trial study". Lipids Health Disease 13 (2014): 191.

12. Ngo-Matip M-E., et al. "Impact of daily supplementation of Spirulina platensis on the immune system of naïve HIV-1 patients in Cameroon: a 12-months single blind, randomized, multicenter trial". Nutrition Journal 21 (2015): 14.

13. Rapport principal EDS-MICS (2011).

14. Moor VJA., et al. "Hypolipidemic effect and activation of Lecithin Cholesterol Acyl Transferase (LCAT) by aqueous extract of Spirulina platensis during toxicological investigation". BMC Nutrition 3.1 (2017): 25.

15. Mouala C. "Use of spirulina supplement for nutritional management of HIV-infected patients: study in Bangui, Central African Republic". Médecine tropicale: revue du Corps de santé colonial 69.1 (2019): 66-70.
16. Balachandran P., et al. "Toll-like receptor 2-dependent activation of monocytes by Spirulina polysaccharide and its immune enhancing action in mice". International Immunopharmacology 6.12 (2006): 1808-1814.

17. Reddy CM., et al. "Selective inhibition of cyclooxygenase-2 by C-phycocyanin, a biliprotein from Spirulina platensis". Biochemical and Biophysical Research Communications 277.3 (2000): 599-603.

18. Mao TK., et al. "Effect of spirulina on the secretion of cytokines from peripheral blood mononuclear cells". Journal of Medicinal Food 3.3 (2000): 135-140.

19. Lee EH., et al. "A randomized study to establish the effects of spirulina in type 2 diabetes mellitus patients". Nutrition Research and Practice 2.4 (2008): 295-300.

20. Tinggi U. "Selenium: its role as antioxidant in human health". Environmental Health and Preventive Medicine 13.2 (2008): 102-108.

21. Salas-Salvadó J., et al. "Effect of a Mediterranean diet supplemented with nuts on metabolic syndrome status: one-year results of the PREDIMED randomized trial". Archives of Internal Medicine 168.22 (2008): 2449-2458.

22. González-Gallego J., et al. "Fruit polyphenols, immunity and inflammation”. British Journal of Nutrition 104 (2010): S15-27.

23. Riss J., et al. "Phycobiliprotein C-phycocyanin from Spirulina platensis is powerfully responsible for reducing oxidative stress and NADPH oxidase expression induced by an atherogenic diet in hamsters". Journal of Agricultural and Food Chemistry 55.19 (2017): 7962-7967.

24. Khan Z., et al. "Nutritional and therapeutic potential of Spirulina". Current Pharmaceutical Biotechnology 6.5 (2005): 373-379.

25. Ch Romay., et al. "C-phycocyanin: a biliprotein with antioxidant, anti-inflammatory and neuroprotective effects". Current Protein and Peptide Science 4.3 (2003): 207-216.

26. Ou Y., et al. "Protective effect of C-phycocyanin against carbon tetrachloride-induced hepatocyte damage in vitro and in vivo". Chemico-biological Interaction 185.2 (2010): 94-100. 
27. Hsiao-WeiChen., et al. "Purification and immunomodulating activity of C-phycocyanin from Spirulina platensis cultured using power plant flue gas". Process Biochemistry 49.8 (2014): 1337-1344.

\section{Assets from publication with us}

- Prompt Acknowledgement after receiving the article

- Thorough Double blinded peer review

- Rapid Publication

- Issue of Publication Certificate

- High visibility of your Published work

Website: www.actascientific.com/

Submit Article: www.actascientific.com/submission.php

Email us: editor@actascientific.com

Contact us: +919182824667 